\title{
A Novel ID-based Authentication Framework with Adaptive Privacy Preservation for VANETs
}

\author{
Huang Lu and Jie Li \\ Department of Computer Science \\ University of Tsukuba \\ Tsukuba Science City, Japan
}

\author{
Mohsen Guizani \\ Qatar University \\ Doha, Qatar
}

\begin{abstract}
In Vehicular Ad hoc Networks (VANETs), authentication is a crucial security requirement to avoid attacks to both inter-vehicle and vehicle-roadside communication. Vehicles have to be prevented from the misuse of their private data and the attacks on their privacy. In this paper, we investigate the authentication and privacy issues in VANETs. We propose a novel ID-based authentication framework with adaptive privacy preservation for VANETs. In this framework, adaptive selfgenerated pseudonyms are used as identifiers instead of realworld IDs. The update of the pseudonyms depends on vehicular demands. The ID-Based Signature (IBS) scheme and the IDBased Online/Offline Signature (IBOOS) scheme are used, for authentication between the Road Side Units (RSUs) and vehicles, as well as authentication among vehicles, respectively. System evaluation has been executed using efficient IBS and IBOOS schemes. It shows that, the proposed authentication framework with privacy preservation is suitable to the VANET environment.

Index Terms-VANETs, pseudonym-based privacy preservation, ID-based authentication, IBS/IBOOS schemes.
\end{abstract}

\section{INTRODUCTION}

A Vehicular Ad hoc Network (VANET) is a technology that employs moving vehicles as nodes in a network to create a mobile network to provide communication among nearby vehicles, as well as between vehicles and nearby fixed Road Side Units (RSUs) |1|. A VANET is considered as a variant form of a Mobile Ad hoc Network (MANET). It turns every participating vehicle into a wireless router or node, allows vehicles in approximately 300 meters range to connect with each other. A VANET thus creates a wide range wireless network for vehicles and RSUs. As mobile wireless devices and wireless networks become increasingly important in recent years, the demand for Vehicle-to-Vehicle (V2V) communication and Vehicle-to-Roadside (V2R) communication increases continuously |1 || 2|. Unlike MANETs, the mobility of vehicles is constrained by predefined roads in VANETs. The vehicle velocities are also restricted on roads according to the speed limits, congestion level, or traffic control mechanisms [2].

A VANET is utilized for a broad range of safety and non-safety applications. It provides value-added services such as location-based services and infotainment applications |1|. Security functions must be supported to prevent potential attacks caused by a vehicle reacting dangerously as a result of receiving erroneous messages. Since access control is generally based on the identity of vehicle, authentication is essential to effective security. Especially, the safety applications require a strong mutual authentication, because most of the safetyrelated messages may contain life-critical information.

We are committed to solving the issues of authentication and privacy in VANETs. There is a number of research work related to the authentication issue in VANETs, and these studies can be classified into two classes: Public Key Infrastructure (PKI) based frameworks and the frameworks using ID-based cryptography. Although many PKI based authentication frameworks have been proposed (e.g., [3], [4], and $[5])$, the system availability will not be pervasive or feasible. Because such frameworks require additional communication to manage the Certificate Revocation Lists (CRLs), which causes heavy overheads. Authentication frameworks using ID-based crypto-system have been proposed to reduce overheads, such as [6], |7|, and [8|. In order to reduce the computational cost in the ID-based Signature (IBS) process for VANETs, the ID-based Online/Offline Signature (IBOOS) scheme is preferable for authentication in VANETs, which is also an attractive solution. An IBOOS scheme [9] increases efficiency of pairing process by separating signing process into an offline phase and an online phase, in which the verification is comparatively more efficient than that of IBS. Therefore, we propose an authentication framework utilizing both the IBS and the IBOOS schemes for better performance. In VANETs, the offline phase can be executed initially at RSUs or vehicles, while the online phase is to be executed in vehicles during $\mathrm{V} 2 \mathrm{~V}$ communication.

In VANETs, vehicles do not want their private information such as name, position, moving route, and user data to be revealed, against illegal tracing and user profiling. The anonymity of identity should be supported in VANETs. The usage of pseudonyms that are intimately linked to an original real-world identity (ID), is a superior solution for privacy preservation to this issue $[10]$. The pseudonym may be generated by the fixed RSUs or the vehicle itself, even can be downloaded from a trusted site periodically, e.g., $|8|$ and [11]. On the other hand, when traffic accidents or certain crimes occur, the identity information has to be revealed by the legal authority to establish the liability of accidents or crimes. VANETs must have a way to validate transmissions and maintain security while retaining privacy.

In this paper, we propose a novel authentication framework with adaptive privacy preservation using ID-based cryptosystem. The primary objective of this work is to provide 
a privacy-preserving authentication mechanism efficiently in terms of the processing time, storage and communication overhead. We utilize self-defined pseudonyms instead of real-world IDs without exposing vehicle privacy. Different from other pre-store schemes (e.g., [8|), an ID pool of regional RSUs is preloaded in each vehicle, because the number of RSUs is comparatively small and does not change frequently. We utilize the IBS scheme for Vehicle-to-Roadside (V2R) authentication and Roadside-to-Vehicle (R2V) authentication, and the IBOOS scheme for Vehicle-to-Vehicle (V2V) authentication. System evaluation shows that, the proposed authentication framework with privacy preservation is efficient and suitable to the VANET environment. It is a novel research work to handle authentication and privacy issues in VANETs using IBOOS, and the main contributions of this paper are as follows.

- We propose an adaptive pseudonym-based scheme, which use self-generated pseudonyms instead of real-world IDs to achieve vehicle privacy and traceability.

- We propose an efficient secure framework to realize authentication with adaptive privacy preservation in VANETs.

- The proposed ID-based authentication framework does not restrict the solution to the existing IBS and IBOOS schemes, thus can also utilize new IBS and IBOOS schemes to achieve improved performance.

The rest parts of this paper is organized as follows. Section II provides the system description and objectives. Section III introduces the preliminary knowledge related to this work. In Section IV, we propose a novel ID-based authentication framework with adaptive privacy preservation for VANETs. Section $\mathrm{V}$ provides analysis on authentication and privacy of the proposed framework, and Section VI evaluates framework performance. In the last section, we conclude the paper.

\section{System Description And Objectives}

This section describes components in the framework and the network structure, as well as the potential threats of VANETs. The objectives are presented afterwards.

\section{A. Network Structure and Components}

A VANET with guaranteed security basically consists of three network components [8] as shown in Figure 1: Road Side Units (RSUs), vehicles (users) and a Regional Trusted Authority (RTA). In this paper, we consider a VANET consisting of a RTA, finite numbered registered RSUs along roads, and a large number of vehicles on or by the roads. This network structure in VANET could be considered as the general urban vehicular communications (UVC) structure. We suppose that, the RSUs are always reliable, while vehicles are vulnerable to being compromised by attackers in UVC. The wireless communication in VANETs can be classified mainly into three types, Vehicle-to-Roadside (V2R) communication, Roadside-to-Vehicle (R2V) communication, and Vehicle-toVehicle (V2V) communication. Other communications are through secure channels, such as inter-RSU communication and RSU-to-RTA communication.

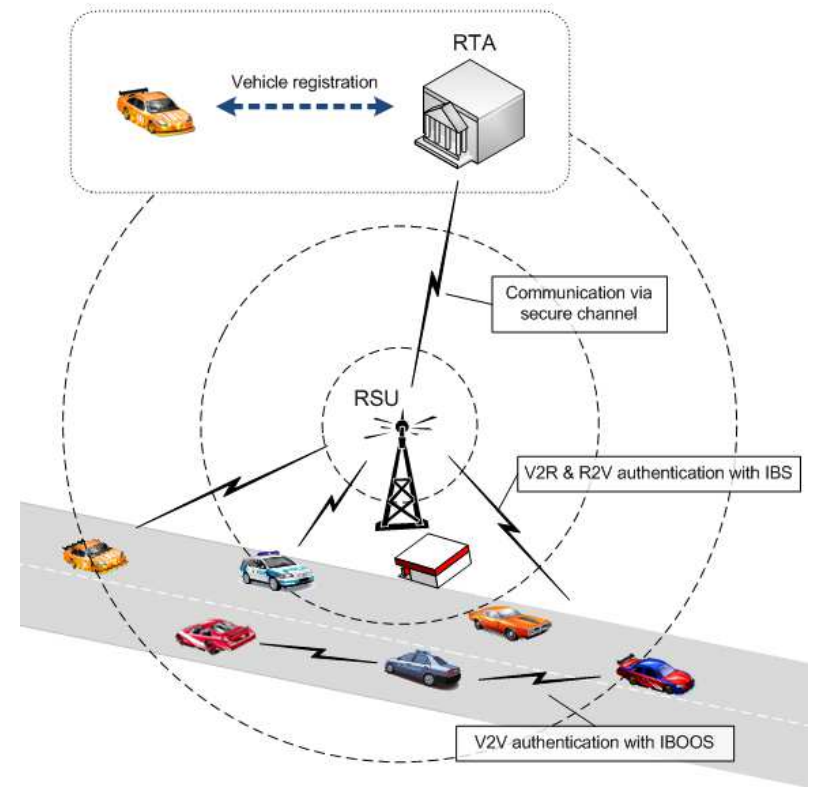

Fig. 1. The illustration of a VANET architecture

Concerning security issues, there are kinds of attacks which threaten the $\mathrm{V} 2 \mathrm{R}$ and $\mathrm{V} 2 \mathrm{~V}$ communication on the road, as we present in the next subsection. The transmission range of an RSU is assumed to be much longer than that of vehicles. All vehicles use symmetric radio channel, and tamper-proof modules (TPMs) are mounted to store sensitive information. The energy of vehicles is adequate and no constrained in a VANET. The candidates for a RTA are automobile manufacturers, an authenticated third party of the state, etc. The main responsibilities of a RTA are shown as follows.

- A RTA generates cryptographic key materials for the RSUs and the vehicles in its region, and delivers these keys to them over secure channels.

- It manages a list of the vehicles of which participations have been revoked, updates the list periodically, and advertises the list to the network to isolate the compromised vehicles.

- If a message sent by a vehicle creates a problem on the road, the RTA is responsible for tracing and identifying the source of the message to resolve the dispute.

- RTAs at different regions have to be cross-certified. Thus vehicles from different regions or different manufacturers can authenticate each other via RTAs.

\section{B. Authentication and Privacy Issues in VANETs}

Once the secure communication has been established for VANETs, many attacks can compromise them. In this Section, we investigate these attacks on authentication and privacy, and explain how they can be performed and the potential consequences.

Attacks on authentication: There are mainly two following attacks related to authentication in VANETs as follows [12].

i. Impersonation attack: The attacker pretends to be another entity. It can be performed by stealing other entity's credential. 
As a consequence, some warnings sent to a specific entity would be sent to an undesired one.

ii. Sybil attack: The attacker uses different identities at the same time. In this way, e.g., a single attacker could pretend vehicles to report the existence of a false bottleneck in traffic.

Attacks on privacy: Attacks on privacy over VANETs are mainly related to illegally gathering sensitive information about vehicles. As there is a relation between a vehicle and its driver, exposure of vehicle's situation could affect its driver privacy |12|.

i. Identity revealing attack: Getting the owner's identity of a given vehicle could put its privacy at risk. Usually, a vehicle's owner is also its driver, so it would simplify getting personal data about that person.

ii. Location tracking attack: The location of a vehicle in a given moment, or the path followed during a period of time is considered as personal data. It allows building that vehicle's profile and, therefore, tracking its driver.

Mechanisms resisting the attacks on both authentication and privacy are required in VANETs. They must satisfy the tradeoff between privacy and utility. We give the definition of authentication with privacy preservation in VANETs below.

Definition 1. In VANETs, authentication with privacy preservation is any process by which a system verifies the approved identity of a vehicle that wishes to access it, whereas the confidential private information will not be disclosed, when that disclosure would cause either embarrassment or distress to the vehicle of reasonable sensitivities.

\section{Objectives}

We endeavor to construct an authentication framework with privacy preservation using ID-based key management for different kinds of communication in VANETs. For authentication, the RTA preloads an ID pool of regional RSUs into a vehicle, and the RSU ID pool does not need to update/replenish unless the RSU ID changes or increases. For the vehicle privacy, we utilize a form of self-defined pseudonyms as real-world IDs without exposing privacy. Therefore, a vehicle can change its pseudonym anytime it wants for privacy preservation. The goal of the proposed authentication framework is to guarantee the privacy-preserving authentication in VANETs.

\section{Preliminaries}

In a VANET, we utilize IBS schemes for authentication between vehicles and an RSU, and IBOOS schemes for authentication among vehicles. In this section, we provide the preliminary knowledge of pairing for ID-based cryptography, as well as the IBS scheme and the IBOOS scheme for authentication, respectively.

\section{A. Pairing for ID-based Cryptography (IBC)}

ID-based crypto-system allows the public key of an entity to be derived from its public identity information such as name, email address, etc, which avoids the use of certificates for public key verification in the conventional PKI. Most of the proposed IBS and IBOOS schemes are based on the Bilinear
Diffie-Hellman Problem (BDHP) and Elliptic Curve Cryptography (ECC) in the pairing domain [1], where the Discrete Logarithm Problem (DLP) for pairing in groups is required to be hard. We briefly review the characteristics of pairing. Randomly select two large primes $p$ and $q$, and let $\mathbb{E} / \mathbb{F}_{p}$ indicate an elliptic curve over the finite field $\mathbb{F}_{p}$. We denote by $\mathbb{G}_{1}$ a $q$-order subgroup of the additive group of points in $\mathbb{E} / \mathbb{F}_{p}$, and $\mathbb{G}_{2}$ a $q$-order subgroup of the multiplicative group in the finite field $\mathbb{F}_{p}^{*}[13]$. The pairing is a mapping $e: \mathbb{G}_{1} \times \mathbb{G}_{1}$ $\rightarrow \mathbb{G}_{2}$, which is a bilinear map if it satisfies the following properties.

1) Bilinear: $\forall P, Q \in \mathbb{G}_{1}$ and $\forall c, d \in \mathbb{Z}_{q}^{*}, e(c P, d Q)=$ $e(P, d Q)^{c}=e(c P, Q)^{d}=e(P, Q)^{c d}$, etc.

2) Non-degeneracy: If $P$ is a generator of $\mathbb{G}_{1}$, then $e(P, P)$ is a generator of $\mathbb{G}_{2}$.

3) Computability: There is an efficient algorithm to compute $e(P, Q)$ in $\mathbb{G}_{2}, \forall P, Q \in \mathbb{G}_{1}$.

Here $\mathbb{Z}_{q}^{*}$ is the multiplicative group consisting of $q-1$ integers. Weil pairing [13| and Tate pairing [14| are the examples of such bilinear mapping, which present comprehensive descriptions of how pairing parameters can be selected for security.

\section{B. ID-based Signature (IBS)}

An IBS scheme consists of four steps including setup, key extraction, signature signing and verification [15].

- Setup: The RTA computes a master key $s$ and public parameters param for the Private Key Generator (PKG) , and gives param to all vehicles.

- Extraction: Given an ID string, the algorithm generates a private key $s e k_{I D}$ associated with the ID using a master key $s$.

- Signature signing: Given a message $M$, time-stamp $t$ and a signing key $\theta$, the algorithm generates a signature $S I G$.

- Verification: Given the $I D, M$ and $S I G$, the verification algorithm outputs "accept" if $S I G$ is valid, and outputs "reject" otherwise.

\section{ID-based Online/Offline Signature (IBOOS)}

An IBOOS scheme consists of five steps including setup, key extraction, offline signing, online signing and verification [16].

- Setup: Same as that in the IBS scheme.

- Extraction: Same as that in the IBS scheme.

- Offline signing: Given public parameters, the algorithm generates an offline signature $S I G^{\text {offline }}$.

- Online signing: From the input of the private key sek $k_{I D}$, the offline signature $S I G^{\text {offline }}$ and a message $M$, the algorithm generates an online signature $S I G^{\text {online }}$ of $M$.

- Verification: Given $I D, M$ and $S I G^{\text {online }}$, the verification algorithm outputs "accept" if $S I G^{\text {online }}$ is valid, and outputs "reject" otherwise.

\section{Proposed Authentication Framework with PRIVACY PRESERVATION FOR VANETS}

This section describes the design of the proposed authentication framework, including protocol initialization and the 
TABLE I

V2R and R2V authentication

$\begin{array}{lllll}\text { Step 1. } & R S U_{r} \Rightarrow * & : & \left\langle I D_{r}, T, \text { adv, nonce }\right\rangle & \text { /* The RSU broadcasts its information. */ } \\ \text { Step 2. } & V_{v} \rightarrow R S U_{r} & : & \left\langle I D_{r}, P S_{v}, T, j o i n, S I G_{v}\left(P S_{v} \| T\right)\right\rangle & \text { / } \text { A vehicle authenticates itself to the RSU. */ } \\ \text { Step 3. } & R S U_{r} \Rightarrow * & : & \left\langle I D_{r}, t, \operatorname{set}(P O I), \text { nonce, } S I G_{R}\left(I D_{r} \| t\right)\right\rangle & \text { /* The RSU broadcasts the POI set. */ }\end{array}$

V2V authentication

Step 1. $\quad V_{v} \rightarrow V_{u} \quad: \quad\left\langle P S_{u}, P S_{v}, t\right.$, nonce, $\left.S I G_{v}^{\text {online }}\left(S I G_{v}^{\text {offline }}\left(P S_{v}\right) \| t\right)\right\rangle \quad / *$ Vehicles authenticate with each other. */

Cross-RSU V2V authentication

$\begin{array}{lllll}\text { Step 1. } & V_{w} \rightarrow V_{u} & : & \left\langle P S_{u}, P S_{w}, t, \text { nonce, } S I G_{w}^{\text {online }}\left(S I G_{w}^{\text {offline }}\left(P S_{w}\right) \| t\right)\right\rangle & \text { /* Vehicle } w \text { authenticates to } u \text {. */ } \\ \text { Step 2. } & V_{u} \rightarrow R S U_{r} & : & \left\langle I D_{r}, P S_{u}, T, q . y ., S I G_{u}\left(P S_{u} \| T\right)\right\rangle & \text { /* } u \text { queries the RSU for authenticity. */ } \\ \text { Step 3. } & R S U_{r} \rightarrow V_{u} & : & \left\langle P S_{u}, I D_{r}, T, q . r ., \text { nonce, } S I G_{R}\left(I D_{r} \| T\right)\right\rangle & \text { /* The RSU replies the query result to } u . * /\end{array}$

- Notation -

\begin{tabular}{|c|c|c|c|}
\hline \multicolumn{4}{|c|}{$*$} \\
\hline & $\Rightarrow, \rightarrow$ & : & The broadcast and unicast communication, respectively. \\
\hline & nonce & $:$ & The number used once, which provides freshness. \\
\hline & $R S U, V$ & : & A road side unit, a vehicle in VANETs, respectively. \\
\hline & $T, t$ & : & $\begin{array}{l}\text { The time-stamp used in } \mathrm{V} 2 \mathrm{R} \text { and } \mathrm{R} 2 \mathrm{~V} \text { authentication, } \\
\text { and the time-stamp used in } \mathrm{V} 2 \mathrm{~V} \text { authentication, respectively. }\end{array}$ \\
\hline & $I D, P S$ & : & The real-world ID and the pseudonym of a vehicle, respectively. \\
\hline & $S I G$ & : & The digital signature generated using IBS. \\
\hline$S I G^{\text {online }}$ & $S I G^{\text {offline }}$ & : & The online and offline digital signature respectively generated using IBOOS. \\
\hline adv, join, set, & $q \cdot y \cdot, q \cdot r$. & : & $\begin{array}{l}\text { The message string types which denote the advertisement, the join_request, } \\
\text { the POI set messages, the query messages, and the query result, respectively. }\end{array}$ \\
\hline
\end{tabular}

pseudonym based authentication framework.

\section{A. Protocol Initialization}

In the proposed framework, RTAs are set in different regions, e.g., a city, a province or a country. Before a vehicle get into the road in a region, the driver first can drive to the RTA for registration. For each vehicle, the RTA publishes the certified domain parameters for authentication, given by: param $=\left(\mathbb{G}_{1}, \mathbb{G}_{2}, q, g, P, s, H\right)$, which are defined in Section IV, and $H$ is the hash function used in the pseudonym generation.

For the purpose of privacy preservation in authentication, vehicles use their self-generated pseudonyms as identifiers instead of real-world IDs, which plays the same role as the identity in the ID-based authentication and secure communications. Meanwhile, the RTA computes all the hash values of registered vehicles, and stores the hash value table. Thus, the RTA can query the real-world ID of a vehicle depending on the certain hash value.

\section{B. Privacy-preserving Authentication}

For privacy preservation, pseudonyms of a vehicle are generated instead of the real-world ID in authentication process. We define the pseudonym of a vehicle as the following form.

$$
\left.P S_{v} \stackrel{\text { def }}{=} \text { RandomNo.\|H(ID }\right) @ H R @ R S U_{C},
$$

where RandomNo. is a random number generated by the Pseudo-Random Number Generator (PRNG). $H\left(I D_{v}\right)$ is a hash value generated from the vehicle's real-world ID. $H R$ denotes the home region where the entity is registered. $R S U_{C}$ denotes the ID of the current corresponding RSU, where the vehicle generates its new pseudonym for secure authentication and communication.

According to the components described in Section II, authentication in VANETs can be divided into three categories, namely Vehicle-to-Roadside (V2R) authentication, Roadsideto-Vehicle (R2V) authentication and Vehicle-to-Vehicle (V2V) authentication. In the proposed framework, RSUs are broadcasting their information periodically, and all the operations at RTAs and RSUs are tamper-proof and being performed trustfully. The proposed authentication framework with privacy preservation operates adaptively, whenever a vehicle wants to newly authenticate itself to others, or update its current pseudonym. Table I shows the operations in the proposed framework.

1) $V 2 R$ and $R 2 V$ Authentication: The V2R and R2V authentication consist of the following three steps. We take an example in Figure 1 to illustrate the authentication between one RSU and a number of vehicles.

Step 1: The RSU broadcasts its information periodically. Therefore, the vehicles in the transmission range can get the RSU's information $\left\langle I D_{r}, T\right.$, adv, nonce $\rangle$. 
Step 2: If a vehicle wants to authenticate itself to others or update its pseudonym in the VANET system, it first uses IBS for V2R authentication. The vehicle unicasts its new pseudonym for the RSU in the message $\left\langle I D_{r}, P S_{v}, T, j o i n, S I G_{v}\left(P S_{v} \| T\right)\right\rangle$, where $I D_{r}$ indicates the destination $R S U_{r}$. After receiving the join_request message from a vehicle, the RSU verifies the signature, and accepts it if the message is authenticated.

Step 3: The RSU first generates the offline signatures $S I G_{v}^{\text {offline }}\left(P S_{v}\right)$ for the vehicle $V_{v}$ based on the pseudonym. The RSU then use IBS for R2V authentication, and broadcasts an allocation set message to all the vehicles in its transmission range for $\mathrm{V} 2 \mathrm{~V}$ communication. The allocation message includes a pseudonym/offline signature/RSU ID (POI) set in the form of $\left(P S_{v} / S I G_{v}^{\text {offline }}\left(P S_{v}\right) / I D_{r}\right)$, attached with a nonce, yet to concatenated with the digital signature $S I G_{r}\left(I D_{r} \| t\right)$. Here $I D_{r}$ denotes the corresponding RSU, where the POI set is generated. All the vehicles in the current RSU's transmission range receive the message, and accept if the signature verification is valid. Then, regarding the acceptable POI according to verification, the vehicle stores the POI set if its storage is possible, otherwise, drops it.

2) V2V Authentication : During the V2V authentication, vehicles can use the received POI set for secure communication. On the authentication with the IBOOS scheme, a vehicle first computes the online digital signature $S I G_{v}^{\text {online }}\left(S I G_{v}^{\text {offline }}\left(P S_{v}\right) \| t\right)$, which depends on the offline signature $S I G_{v}^{\text {offline }}$ and the time stamp $t$. Then the vehicle can use the online signature for the $\mathrm{V} 2 \mathrm{~V}$ authentication, as shown in Step 4.

3) Cross-RSU V2V Authentication: The cross-RSU V2V authentication is required when a vehicle receives an authentication message from another vehicle, whose pseudonym does not appear in its storage. In this case, the vehicle can query the RSU, which consists of the following three steps.

Step 1: We take an example to illustrate this approach, where vehicle $w$ is aiming to authenticate with vehicle $u$ with its online $S I G_{w}^{\text {online }}$.

Step 2: On the receiving authentication message from $w$, the vehicle $u$ checks its storage for the pseudonym and the POI set of $w$. If the information does not appear in vehicle $u$ 's storage, vehicle $u$ transmits its query message $q . y$. of authenticity to the nearest RSU, which includes the POI set of vehicle $w$ in the form of $\left(P S_{w} / S I G_{w}^{\text {offline }}\left(P S_{w}\right) / I D_{r}\right)$, signed with the IBS $S I G_{u}$.

Step 3: After receiving the queried message, the current RSU queries other RSUs or the RTA via secure channels to check if the POI set is authenticated. Afterwards, the current RSU replies the query result q.r. back to the querying vehicle $u$, whether or not the POI set is authenticated.

4) Cross-region Authentication: When a vehicle enters a new region, first it has to go to the current RTA for registration. At a RTA, a vehicle can update or replenish its RSU pool and the certified domain parameters for authentication.

\section{Implementation of Proposed Framework}

There are many IBS and IBOOS schemes available for the proposed framework, mainly based on ECC and RSA signatures. Verifying RSA signature is efficient for a vehicle [17], and we can set small verification exponents. However, RSA based signatures are large, resulting in a considerably increased message size. ECC based signatures are equally useful for signing and verification of messages and have short signature sizes. Therefore, for VANETs, ECC based signatures are considered more efficient than RSA signatures. To exemplify the proposed authentication framework, we select the most secure and efficient ECC based signature schemes from the available IBS and IBOOS schemes. An IBS scheme given in $[18]$ is preferable for $\mathrm{V} 2 \mathrm{R}$ and $\mathrm{R} 2 \mathrm{~V}$ authentication, security of this signature scheme depends on ECC and DLP. While as an IBOOS schemes given in |19| is preferable to satisfy V2V broadcast authentication scheme, where the offline signature can be securely reused to sign more than one message, and its security also depends on DLP.

\section{Analysis on Authentication and PRivacy}

The proposed framework primarily attempts to provide authentication and privacy preservation in VANETs, and resolve the conflicts between them. According to the Definition 1 in Section III, we analyze authentication and privacy preservation respectively as follows.

1) Authentication: In the proposed framework, authentication is guaranteed by digital signatures, which bound a message to a pseudonym and consequently the corresponding identity. In addition, digital signature also guarantees nonrepudiation of the signer by querying the vehicle's real-world ID from the RTA.

2) Privacy Preservation: The adoption of pseudonyms in VANET communications conceals the real-world identity of vehicles such that peer vehicles and RSUs cannot identify the sender of a specific message, meanwhile is still able to authenticate the sender. By frequently updating the pseudonyms during communication, the proposed framework successfully defends legitimate vehicles against location tracing and user profiling.

In action, the vehicle will interact primarily with the RSUs for Internet access, or with peer vehicles for information exchange. Since the secure interactions with the RTA or RSUs are proposed in the proposed framework, we can safely claim that attackers cannot link pseudonyms with the identity to compromise the privacy of an honest vehicle. By adjusting the length of the update period, the vehicle can control the achievable privacy by limiting the RSUs' broadcast of POI sets. As a result, except for the cases where identities must be revealed to the RTA, no other entities in the proposed system can compromise the privacy of honest vehicle users.

\section{Vi. Performance Evaluation}

This section evaluates the performance and efficiency of the proposed authentication framework in terms of storage requirement and computation efficiency. 


\section{A. Storage Requirement}

In the proposed framework, the storage requirements on RTAs and RSUs are not stringent since these entities are distributed and resource-free in nature for UVC in VANETs. We are mainly concerned with the storage cost in vehicles with two respects, the information necessary for cryptographic parameters, and the number of POI sets for $\mathrm{V} 2 \mathrm{~V}$ authentication.

In order to gain a high security level in the proposed framework, we adopt the parameters chosen by [4], which results in a security level similar to 2048-bit RSA and a total storage space of $4.2 \mathrm{M}$ bytes. In this scheme, the ECC based PKI was adopted, which is well-known for its efficient storage and communication performance compared to the RSA based PKI.

\section{B. Computation Efficiency}

This part gives an estimation of efficiency on applying the proposed framework for VANETs. Although the computationally intensive pairing operations are not involved in conventional PKI, we believe that the ID-based crypto-system based on pairings is highly suitable, especially in the VANET environment.

TABLE II

COMPARISON OF EFFICIENCY USING DIFFERENT CRYPTOGRAPHIES

\begin{tabular}{|c|c|c|c|c|}
\hline Scheme & Signature & $\begin{array}{c}\text { Energy Cost } \\
\text { (verification) } \\
(\mathrm{mW})\end{array}$ & $\begin{array}{c}\text { Computation } \\
\text { time (online) } \\
(\mathrm{ms})\end{array}$ & $\begin{array}{c}\text { Message } \\
\text { size } \\
(\text { byte })\end{array}$ \\
\hline$|18|$ & IBS & 26.96 & 18.10 & $125+$ Signature \\
\hline$|20|$ & IBS & 71.90 & 14.10 & $42+$ Signature \\
\hline$|19|$ & IBOOS & negligible & negligible & 84 \\
\hline$|21|$ & IBOOS & 5.62 & 0.19 & $64+$ Signature \\
\hline
\end{tabular}

Consider that the well-known Tate pairing is used for the basic pairing operation of the IBS scheme. It is shown in [18] that the time taken for computing a Tate pairing is $20 \mathrm{~ms}$, in the underlying base field of $F_{p}$ (where $|p|=512$-bit), which has a similar level of security to 1024-bit RSA. The proposed V2V authentication scheme using IBOOS [19| allows the secure reuse of the offline signature, computed by the RSU. The only cost a vehicle bears in message signing is the cost of the online phase which requires two point multiplications in offline phase. This online phase only consists of integer addition and multiplication operations, which are very efficient for vehicles in terms of time and energy consumption. Therefore, the time and energy cost of the online phase is almost negligible. In Table II, we show the efficiency of authentication with a comparison of using different cryptographic schemes. Here, the computation time indicates the verification time used for the signature in IBS schemes and the online signature in IBOOS schemes. Energy cost indicates the energy assumed for signature verification at the receiving vehicle.

\section{CONCLUSiOn AND Future Work}

In this paper, an ID-based authentication framework with adaptive privacy preservation has been proposed for UVC in VANETs, which utilizes IBS and IBOOS schemes for authentication, and a pseudonym-based scheme for privacy preservation. One of the advantages of this framework is its reusability, which means that, it can also be reused with new IBS and IBOOS schemes for security and performance improvements. In the future, we intend to focus on providing a complete security framework, which would enable other security requirements such as data consistency, non-repudiation and availability.

\section{REFERENCES}

[1] S. Zeadally, R. Hunt, Y.-S. Chen, A. Irwin, and A. Hassan, "Vehicular ad hoc networks (VANETS): status, results, and Challenges," Telecommunication Systems (Online First), pp. 1-25, 2010.

[2] F. Li and Y. Wang, "Routing in vehicular ad hoc networks: A survey," IEEE Vehicular Technology Magazine, vol. 2, no. 2, pp. 12-22, 2007.

[3] "IEEE 1609 Family of Standards for Wireless Access in Vehicular Environments (WAVE)," U.S. Department of Transportation, 2009. Available: IEEEStandards

[4] M. Raya and J. Pierre, "Securing vehicular ad hoc networks," Journal of Computer Security, vol. 15, no. 1, pp. 39-68, 2007.

[5] N.-W. Wang, Y.-M. Huang, and W.-M. Chen, "A novel secure communication scheme in vehicular ad hoc networks," Computer Communications, vol. 31, pp. 2827-2837, 2008.

[6] P. Kamat, A. Baliga, and W. Trappe, "Secure, pseudonymous, and auditable communication in vehicular ad hoc networks," Security and Communication Networks, vol. 1, no. 3, pp. 233-244, 2008.

[7] X. Lin, X. Sun, P.-H. Ho, and X. Shen, "GSIS: A Secure and PrivacyPreserving Protocol for Vehicular Communications," IEEE Transactions on Vehicular Technology, vol. 56, no. 6, pp. 3442-3456, 2007.

[8] J. Sun, C. Zhang, Y. Zhang, and Y. Fang, "An Identity-Based Security System for User Privacy in Vehicular Ad Hoc Networks," IEEE Transactions on Parallel and Distributed Systems, vol. 21, no. 9, pp. 1227-1239, 2010 .

[9] S. Even, O. Goldreich, and S. Micali, "On-Line/Off-Line Digital Signatures,” in Adv. in Cryptology - CRYPTO'89, LNCS, vol. 435, 1990.

[10] H. Dok, H. Fu, R. Echevarria, and H. Weerasinghe, "Privacy Issues of Vehicular Ad-Hoc Networks," Int'l Journal of Future Generation Communication and Networking, vol. 3, no. 1, pp. 17-32, 2010.

[11] M. Gerlach and F. Guttler, "Privacy in VANETs using Changing Pseudonyms - Ideal and Real," in Proc. of IEEE 65th Vehicular Technology Conference, VTC 2007-Spring, 2007.

[12] J. M. D. Fuentes, A. I. González-Tablas, and A. Ribagorda, "Overview of Security Issues in Vehicular Ad-hoc Networks," in Handbook of Research on Mobility and Computing: Evolving Technologies and Ubiquitous Impacts. IGI Global, 2011, pp. 894-911.

[13] D. Boneh and M. Franklin, "Identity-Based Encryption from the Weil Pairing," in Adv. in Cryptology - CRYPTO 2001, LNCS, vol. 2139, 2001.

[14] P. Barreto, H. Kim, B. Lynn, and M. Scott, "Efficient Algorithms for Pairing-Based Cryptosystems," in Adv. in Cryptology - CRYPTO 2002, LNCS, vol. 2442, 2002.

[15] A. Shamir, "Identity-Based Cryptosystems and Signature Schemes," in Adv. in Cryptology - CRYPTO'84, LNCS, vol. 196, 1985.

[16] A. Shamir and Y. Tauman, "Improved Online/Offline Signature Schemes," in Adv. in Cryptology - CRYPTO 2001, LNCS, vol. 2139, 2001.

[17] N. Gura, A. Patel, A. Wander, H. Eberle, and S. C. Shantz, "Comparing Elliptic Curve Cryptography and RSA on 8-bit CPUs," in Cryptographic Hardware and Embedded Systems - CHES 2004, LNCS, vol. 3156, 2004.

[18] D. Boneh, B. Lynn, and H. Shacham, "Short Signatures from the Weil Pairing," Journal of Cryptology, vol. 17, pp. 297-319, 2004.

[19] S. Xu, Y. Mu, and W. Susilo, "Efficient Authentication Scheme for Routing in Mobile Ad Hoc Networks," in Embedded and Ubiquitous Computing - EUC 2005 Workshops, LNCS, vol. 3823, 2005.

[20] C. Zhang, R. Lu, X. Lin, P.-H. Ho, and X. Shen, "An Efficient IdentityBased Batch Verification Scheme for Vehicular Sensor Networks," in Proc. of IEEE 27th Conference on Computer Communications, INFOCOM 2008, 2008.

[21] Q. Ren, Y. Mu, and W. Susilo, "Mitigating phishing with ID-based online/offline authentication," in Proc. of the sixth Australasian conference on Information security, AISC'08, 2008. 